\title{
DINÂMICA POPULACIONAL DO BICHO-MINEIRO E DE SEUS INIMIGOS NATURAIS EM CAFEEIROS ORGÂNICO E CONVENCIONAL ADENSADOS
}

\author{
Carvalho Carlos Ecole ${ }^{1}$, Michelle Vilela ${ }^{2}$, Jair Campos Moraes ${ }^{3}$, Rogério Antônio Silva ${ }^{4}$ \\ ${ }^{1}$ Agrônomo, DSc em Agronomia/Entomologia - Instituto de Investigação Agrária de Moçambique (IIAM) Direcção de \\ Agronomia \& Recursos Naturais (DARN) - Av. das FPLM, 2698 - P.O.Box 3658 - Mavalane - Maputo, Moçambique - \\ ccecole@hotmail.com \\ ${ }^{2}$ Agronôma, Doutoranda em Agronomia/Entomologia (UFLA) - Universidade Federal de Lavras - Caixa Postal 3037 - CEP: \\ 37200-000 - Lavras, MG - mimi_vilela@yahoo.com.br \\ ${ }^{3}$ Agrônomo, DSc Professor/Pesquisador no Departamento de Entomologia da Universidade Federal de Lavras - Caixa Postal 3037 \\ - CEP: 37200-000 - Lavras, MG - jcmoraes@ufla.br \\ ${ }^{4}$ Agrônomo, DSc em Agronomia/Entomologia - IMA/EPAMIG-CTSM/EcoCentro - Unidade Regional Epamig Sul de Minas - \\ Caixa Postal 176 - CEP: 37200-000 - Lavras, MG - rogeriosilva@epamig.br
}

\section{RESUMO}

A presente pesquisa foi realizada em dois talhões de 2,0 ha de cafeeiros sob plantio adensado $(2,0 \times 1,0 \mathrm{~m})$, cultivar Acaiá/IAC-474-19 : um sob cultivo orgânico e outro convencional, com cinco anos de idade em Santo Antônio do Amparo, MG. Estudou-se a dinâmica populacional do bicho-mineiro Leucoptera coffeella (Guérin-Mèneville \& Perrottet, 1842) (Lepidoptera: Lyonetiidae) e seus inimigos naturais. Avaliaram-se a porcentagem de folhas minadas, a porcentagem de minas predadas por vespas, número de lagartas do bicho-mineiro vivas, número de pupas formadas/60 folhas coletadas na amostragem e a porcentagem de parasitismo total. Os dados referentes a cada avaliação e sistema de cultivo foram submetidos à análise de variância e ao teste de médias de Scott-Knott ( $\mathrm{p} \leq 0,05)$. Observou-se que, em 1999, o cultivo de café orgânico apresentou a maior porcentagem de folhas minadas pelo bicho-mineiro $(45,3 \%)$, de minas predadas por vespas $(8,0 \%)$, maiores números de lagartas vivas e de pupas formadas, e maior porcentagem de parasitismo total $(65,5 \%)$, em relação ao sistema convencional (11,2\% de folhas minadas e $36,1 \%$ de parasitismo total). Esta situação se inverteu para a porcentagem de folhas minadas e número de lagartas vivas em 2000 e 2001. Não foram detectados parasitoides de ovos de bicho-mineiro em nenhum dos sistemas de cultivo. As espécies de parasitoídes mais abundantes foram Orgilus niger, Centistidea striata, Stiropius reticulatus (Hymenoptera: Braconidae) e Horismenus sp. (Hymenoptera: Eulophidae).

Palavras-chave: Coffea arabica, Leucoptera coffeella, parasitismo, sistemas de cultivo

\section{POPULATION-DYNAMICS OF THE COFFEE LEAF-MINER AND ITS NATURAL ENEMIES IN DENSE ORGANIC AND CONVENTIONAL COFFEE CROPS}

\section{ABSTRACT}

The present research was carried out in a two planting fields of 2.0 ha of coffee crops under dense plantation $(2.00 \times 1.00 \mathrm{~m})$, cultivar of Acaiá/IAC-474-19 : one under organic crop and the other in conventional crop, with five years of age, in Santo Antônio do Amparo, State of Minas Gerais, Brazil. The population dynamics of the coffee leaf-miner Leucoptera coffeella (Guérin-Mèneville \& Perrottet, 1842) (Lepidoptera: Lyonetiidae) and its natural enemies were studied. The percentage of mined leaves, mines preyed by wasps, the number of live caterpillars of the coffee leaf-miners, the number of pupae in sixty leaves collected in the sample and total 
percentage of parasitism were assessed. The data referring to each assessment and crop system were submitted to the variance analysis and to the grouped means test of Scott \& Knott $(\mathrm{p} \leq 0.05)$. It was observed in 1999 that the organic coffee crop showed a higher percentage of leaves mined by the coffee leaf-miner $(45.3 \%)$, mines preyed by wasps $(8.0 \%)$, a higher number of live caterpillars and pupae, and a higher percentage of total parasitism $(65.5 \%)$, in relation to the conventional crop system ( $11.2 \%$ of mined leaves and $36.1 \%$ of total parasitism). This situation was inverted for the percentage of mined leaves and the number of live caterpillars in 2000 and 2001. No egg parasitoids of the coffee leaf-miner were detected in any of the crop systems. The most abundant species of larval parasitoids were Orgilus niger, Centistidea striata, Stiropius reticulatus (Hymenoptera: Braconidae) and Horismenus sp. (Hymenoptera: Eulophidae).

Key words: Coffea arabica, Leucoptera coffeella, parasitism, crop systems

\section{INTRODUÇÃO}

A importância do café na economia do Brasil é inquestionável. O país é o maior produtor e exportador desta commodity e o segundo maior mercado consumidor no mundo, atrás somente dos Estados Unidos. A produção brasileira em 2008 foi de 46 milhões de sacas; as exportações foram de 29,5 milhões de sacas, e o consumo interno de 17,66 milhões de sacas (ABIC, 2009).

Entretanto, prejuízos econômicos podem ser provocados por insetos-praga como o bicho-mineiro, Leucoptera coffeella (Guérin-Mènéville \& Perrottet, 1842) (Lepidoptera: Lyonetiidae), que sob determinadas condições assume o "status" de praga-chave (Souza et al., 1998), e o conhecimento acerca dos níveis populacionais desta praga, da diversidade de parasitoides e dos índices de parasitismo natural são fundamentais para a tomada de decisão na adoção de estratégias de manejo (Melo et al., 2007).

No controle do bicho-mineiro têm sido utilizados inseticidas os quais, não raro, causam desequilíbrios ecológicos por eliminação de insetos benéficos, ressurgência e surtos populacionais da praga e, principalmente, intoxicação nos aplicadores (Guedes \& Fragoso, 1999; Fragoso et al., 2002).

Os sistemas agrícolas diversificados podem reduzir a incidência de insetos-praga e/ou aumentar a atividade de inimigos naturais (Aguiar-Menezes, 2004). O cultivo orgânico é uma alternativa viável e evita a utilização e os efeitos prejudiciais causados pelo uso indiscriminado de produtos químicos (Theodoro et al., 2002; Ecole et al., 2010), além de preservar populações de parasitoides em cafeeiros cultivados tanto em locais com ou sem arborização (AguiarMenezes et al., 2008).

Entretanto, existem poucas informações sobre a dinâmica populacional do bicho-mineiro e de seus inimigos naturais em sistemas de cultivo orgânico, nos quais são utilizadas práticas racionais e de baixo impacto. Assim, o objetivo neste trabalho foi avaliar o efeito dos sistemas de cultivo dos cafeeiros, orgânico e convencional adensados, na dinâmica populacional de $L$. coffeella e de seus inimigos naturais.

\section{MATERIAL E MÉTODOS}

A pesquisa foi desenvolvida na Fazenda Cachoeira, município de Santo Antônio do Amparo, MG, situada a $1.000 \mathrm{~m}$ de altitude, coordenadas geográficas de

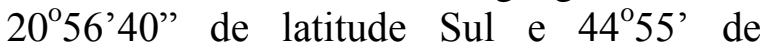
longitude Oeste. O clima da região, de acordo com a classificação de Koppen, enquadra-se no tipo Cwa, que corresponde ao clima tropical e subtropical chuvoso (mesotérmico) com inverno seco e verão chuvoso. A altitude da região varia de 600 a 
$1.200 \mathrm{~m}$, sendo o relevo ondulado a montanhoso. As precipitações médias anuais são de 1.400 a $1.700 \mathrm{~mm}$. O inverno dura de dois a quatro meses secos como na região sudeste do Brasil, todavia com déficit hídrico estreitado a 10 a $30 \mathrm{~mm}$ e evapotranspiração potencial de 800 a 850 mm anuais (Antunes, 1986).

$\mathrm{O}$ experimento foi realizado em dois talhões de 2,0 ha de cafeeiro em plantio adensado $(2,0 \times 1,0 \mathrm{~m}$, espaçamento entre linhas e plantas, respectivamente), variedade Acaiá/IAC-474-19 com cinco anos de idade. Um desses talhões conduzido no sistema orgânico localizava-se na Fazenda Cachoeira e o outro, no sistema convencional, na Fazenda Taquaril, pertencente ao senhor Armando Leite Nave, localizada a $3 \mathrm{~km}$ da anterior.

No sistema orgânico, a adubação foi feita com base na compostagem de materiais orgânicos, como a casca de café, esterco de curral, chorume, capim picado, mamona picada e triturada (restos de cultura) e micronutrientes na forma de sais. O mato foi recepado e enleirado no cultivo, as doenças fúngicas foram controladas com fungicidas recomendados pelas certificadoras como a "calda viçosa" ou por meio de pulverizações de soluções com micronutrientes. No sistema convencional, o produtor utilizava as práticas corriqueiras, como as capinas nos espaçamentos entre plantas e aplicações de herbicidas nas entrelinhas para o controle de infestantes. Para o controle de doenças utilizava produtos cúpricos, efetuava adubações minerais e aplicação de cartap e imidacloprid para o controle do bichomineiro e endossulfan para o controle da broca-do-café. Foram feitas quatro aplicações desses compostos durante o período do experimento, sendo uma no segundo semestre de 1999, duas aplicações em 2000 e uma em 2001.

Para a instalação do experimento foram demarcadas quatro parcelas/talhão, constituídas por quatro fileiras, com dez plantas/fileira, num total de 40 plantas/parcela, sendo a parcela útil as 16 plantas centrais. Adotou-se o delineamento inteiramente casualizado, em parcelas subdivididas, com dois sistemas de cultivo (tratamentos) e quatro repetições. Realizaram-se três avaliações no $2^{\underline{0}}$ semestre de 1999, sete e cinco avaliações, em 2000 e 2001, respectivamente.

As amostragens foram feitas coletando-se a terceira ou quarta folhas de ramos do terço médio (quatro folhas/planta, totalizando 60 folhas/parcela). As folhas foram conservadas em sacos de papel, colocadas em caixa de isopor, levadas ao Laboratório de Manejo Integrado de Pragas da UFLA, onde foram inspecionadas sob lupa estereoscópica. As folhas com sinais da praga foram lavadas em solução de hipoclorito de sódio a 5\% para eliminação de contaminantes. Depois, foram imersas pelo pedúnculo em solução de benziladenina contida em caixas de Gerbox e mantidas em câmara climatizada $25 \pm 1{ }^{\circ} \mathrm{C}$, umidade relativa de $65 \pm 5 \%$ e fotofase de 12 horas, por 25 a 30 dias, para observação da emergência do bicho-mineiro ou dos parasitoides.

As características avaliadas foram: porcentagem de folhas minadas, porcentagem de minas predadas por vespas, número de lagartas do bicho-mineiro vivas, número de pupas formadas/60 folhas coletadas na amostragem e a porcentagem de parasitismo total (para as quatro espécies de parasitoides mais freqüentes nas coletas). Também foram obtidos os valores de precipitação pluvial e de temperatura no período correspondente. Os dados referentes a cada avaliação e sistema de cultivo foram submetidos à análise de variância e ao teste de médias de Scott \& Knott $(p \leq 0,05)$ (Scott \& Knott, 1974). Os espécimens dos parasitoides foram identificados pela Dra. Angélica Maria Penteado-Dias, da Universidade Federal de São Carlos, em São Carlos, estado de São Paulo. 


\section{RESULTADOS E DISCUSSÃO}

No ano de 1999 o cafeeiro em cultivo orgânico apresentou maiores porcentagens de folhas minadas pelo bicho-mineiro $(45,3 \%)$, de minas predadas por vespas $(8,0 \%)$, números de lagartas vivas e de pupas formadas da praga e porcentagem de parasitismo total por Braconidae e Eulophidae $(65,5 \%)$, para cada mês do ano em que foi feita a avaliação, do que $o$ sistema convencional com $11,2 \%$ de folhas minadas, $0,8 \%$ de minas predadas por vespas e $36,1 \%$ de parasitismo total (Tabelas 1 a 3). Os índices de predação de lagartas de $L$. coffeella observados nesse ano foram baixos (Tabela 1) em relação aos relatados por outras pesquisas para o Sul de Minas, em cafeeiros não adensados e convencionais em que normalmente são aplicados produtos fitossanitários (Reis \& Souza, 1998; Reis Jr. et al., 2000).

Tabela 1. Folhas minadas (\%) por Leucoptera coffeella e minas predadas (\%) por vespas em função do sistema de cultivo do cafeeiro e da época de avaliação. Santo Antônio do Amparo, MG.

\begin{tabular}{lcccc}
\hline \multirow{2}{*}{ Mês } & \multicolumn{3}{c}{ Folhas minadas (\%) } & \multicolumn{3}{c}{ Minas predadas (\%) } \\
\cline { 2 - 5 } & \multicolumn{5}{c}{ Orgânico } & Convencional & Orgânico \\
\cline { 2 - 5 } Junho & Convencional & $42,0 \mathrm{aB}$ & $0,0 \mathrm{bA}$ & $4,0 \mathrm{aB}$ \\
Agosto & $1,5 \mathrm{bB}$ & $26,0 \mathrm{aC}$ & $0,0 \mathrm{bA}$ & $4,0 \mathrm{aB}$ \\
Novembro & $0,5 \mathrm{bC}$ & $68,0 \mathrm{aA}$ & $2,5 \mathrm{bA}$ & $16,0 \mathrm{aA}$ \\
\hline \multicolumn{5}{c}{2000} \\
\hline Janeiro & $31,5 \mathrm{bA}$ & $35,5 \mathrm{aA}$ & $1,5 \mathrm{bA}$ & $16,5 \mathrm{aA}$ \\
Abril & $20,5 \mathrm{aB}$ & $0,0 \mathrm{aB}$ & $0,5 \mathrm{aA}$ & $0,0 \mathrm{aB}$ \\
Maio & $3,0 \mathrm{aC}$ & $22,0 \mathrm{aA}$ & $1,5 \mathrm{aA}$ & $8,0 \mathrm{aB}$ \\
Agosto & $6,0 \mathrm{aC}$ & $14,0 \mathrm{aA}$ & $2,0 \mathrm{aA}$ & $2,5 \mathrm{aB}$ \\
Setembro & $31,5 \mathrm{aB}$ & $27,5 \mathrm{bA}$ & $2,5 \mathrm{aA}$ & $2,0 \mathrm{aB}$ \\
Outubro & $58,5 \mathrm{aA}$ & $5,5 \mathrm{bB}$ & $8,5 \mathrm{aA}$ & $0,5 \mathrm{bB}$ \\
Novembro & $46,5 \mathrm{aA}$ & $5,0 \mathrm{bB}$ & $0,5 \mathrm{aA}$ & $1,5 \mathrm{aB}$ \\
Dezembro & $34,5 \mathrm{aB}$ & $0,0 \mathrm{aB}$ & $0,0 \mathrm{aA}$ & $0,0 \mathrm{aB}$ \\
\hline & $3,0 \mathrm{aC}$ & & & \\
\hline Janeiro & & $0,0 \mathrm{aB}$ & - & - \\
Fevereiro & $0,0 \mathrm{aB}$ & $0,0 \mathrm{aB}$ & - & - \\
Abril & $0,0 \mathrm{aB}$ & $0,5 \mathrm{aB}$ & - & - \\
Maio & $0,0 \mathrm{aB}$ & $0,0 \mathrm{aA}$ & - & - \\
Junho & $5,0 \mathrm{aA}$ & $6,5 \mathrm{aA}$ & - & - \\
\hline
\end{tabular}

* Médias seguidas pela mesma letra minúscula na linha e maiúscula na coluna não diferem entre si pelo teste de Scott-Knott $(p \leq 0,05)$. 
Tabela 2. Número de lagartas vivas e de pupas formadas de Leucoptera coffeella/60 folhas coletadas, em função dos sistemas de cultivo do cafeeiro e da época de avaliação. Santo Antônio do Amparo, MG.

\begin{tabular}{|c|c|c|c|c|}
\hline \multirow{3}{*}{ Mês } & \multicolumn{2}{|c|}{ № de lagartas vivas/ 60 folhas } & \multicolumn{2}{|c|}{ № de pupas/ 60 folhas } \\
\hline & \multicolumn{4}{|c|}{1999} \\
\hline & Convencional & Orgânico & Convencional & Orgânico \\
\hline Junho & $0,7 \mathrm{bA}$ & $4,0 \mathrm{aB}$ & $0,3 \mathrm{bB}$ & $4,3 \mathrm{aB}$ \\
\hline Agosto & $0,7 \mathrm{bA}$ & $4,0 \mathrm{aB}$ & $0,3 \mathrm{bB}$ & $11,0 \mathrm{aB}$ \\
\hline \multirow[t]{2}{*}{ Novembro } & $2,7 \mathrm{bA}$ & $16,0 \mathrm{aA}$ & $4,5 \mathrm{bA}$ & $15,8 \mathrm{aA}$ \\
\hline & \multicolumn{4}{|c|}{2000} \\
\hline Janeiro & $0,3 \mathrm{aC}$ & $1,0 \mathrm{aC}$ & $3,5 \mathrm{aC}$ & $3,3 \mathrm{aC}$ \\
\hline Abril & $0,3 \mathrm{aC}$ & $0,0 \mathrm{aC}$ & - & - \\
\hline Maio & $5,0 \mathrm{aB}$ & $5,0 \mathrm{aB}$ & $11,0 \mathrm{aB}$ & $10,8 \mathrm{aB}$ \\
\hline Agosto & $15,0 \mathrm{aB}$ & $5,5 \mathrm{bB}$ & $23,5 \mathrm{aA}$ & $11,3 \mathrm{bA}$ \\
\hline Setembro & $27,3 \mathrm{aA}$ & $15,0 \mathrm{bA}$ & $11,0 \mathrm{aB}$ & $9,3 \mathrm{bB}$ \\
\hline Outubro & $0,0 \mathrm{aC}$ & $3,3 \mathrm{aC}$ & $8,8 \mathrm{aB}$ & $7,8 \mathrm{aB}$ \\
\hline Novembro & $7,3 \mathrm{aC}$ & $0,5 \mathrm{bC}$ & $8,0 \mathrm{aB}$ & $2,5 \mathrm{bC}$ \\
\hline \multirow[t]{2}{*}{ Dezembro } & $0,0 \mathrm{aC}$ & $0,0 \mathrm{aC}$ & $0,0 \mathrm{aD}$ & $0,0 \mathrm{aD}$ \\
\hline & \multicolumn{4}{|c|}{2001} \\
\hline Janeiro & - & - & $0,0 \mathrm{aC}$ & $0,0 \mathrm{aB}$ \\
\hline Fevereiro & - & - & $0,0 \mathrm{aC}$ & $0,0 \mathrm{aB}$ \\
\hline Abril & - & - & $0,0 \mathrm{aC}$ & $0,0 \mathrm{aB}$ \\
\hline Maio & - & - & $29,5 \mathrm{aA}$ & $5,3 \mathrm{bA}$ \\
\hline Junho & - & - & $15,0 \mathrm{aB}$ & $9,8 \mathrm{aA}$ \\
\hline
\end{tabular}

* Médias seguidas pela mesma letra minúscula na linha e maiúscula na coluna não diferem entre si, pelo teste de Scott-Knott $(p \leq 0,05)$.

Contudo, os índices de parasitismo de lagartas do bicho-mineiro observados no mesmo ano e para o sistema orgânico foram superiores a $40 \%$, o que discorda com observações de pesquisadores que já estudaram o controle biológico natural dessa praga no Brasil e em cultivo convencional (Parra et al., 1981; Reis \& Souza, 1983; Reis Jr. et al., 2000), confirma, portanto, o pressuposto de que nos sistemas de cultivo convencional, a seletividade ecológica, conseguida para vespas predadoras (aplicação dos inseticidas no momento em que as vespas estão ausentes do cultivo), pode não ocorrer para endoparasitoides já que estes não têm como escapar do efeito deletério dos pesticidas ou da predação por vespa, por se desenvolverem no interior da lagarta do bicho-mineiro (Ecole et al., 2010).
No segundo ano de observações (2000), embora as secas do Sul de Minas tenham persistido, os dois sistemas de cultivo do cafeeiro não diferiram quanto à porcentagem de folhas minadas pelo bicho-mineiro nos quatro primeiros meses (Tabelas 1 a 3). A partir do quinto mês o sistema orgânico teve menor porcentagem de folhas minadas $(27,5 \%)$ em relação ao convencional $(58,5 \%)$ e menor número de lagartas vivas (agosto, setembro e novembro); em dezembro não foram observadas lagartas vivas e nem lesões do bicho-mineiro em ambos os sistemas de cultivo do cafeeiro. Por ser um período de precipitações elevadas em Santo Antônio do Amparo, há possibilidade da chuva ter reduzido intensamente a população de lagartas do bicho-mineiro (Figura 1). 


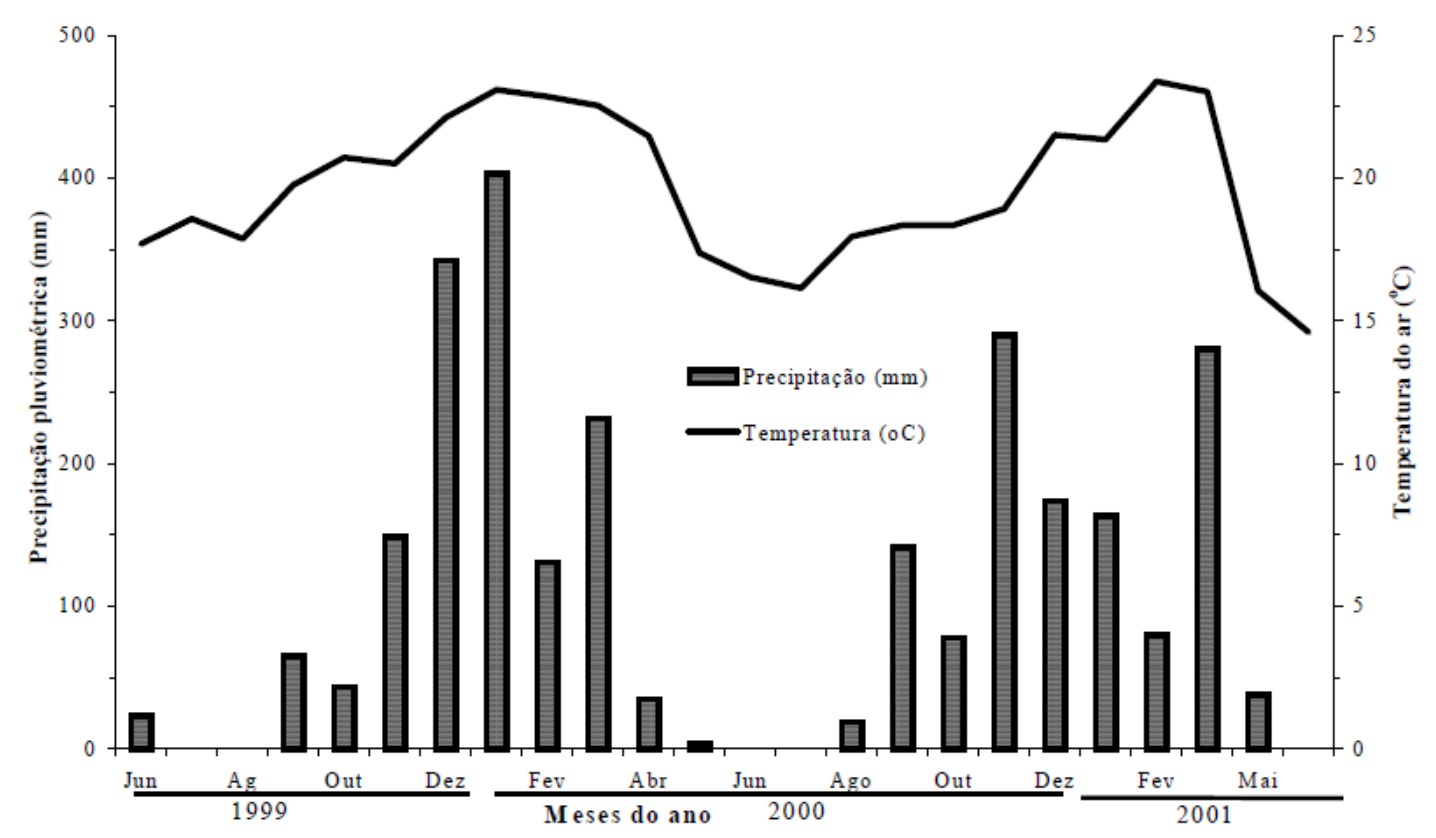

Figura 1. Valores médios de temperatura do ar $\left({ }^{\circ} \mathrm{C}\right)$ e total de precipitação pluviométrica (mm) de junho de 1999 a junho de 2001, Santo Antônio do Amparo, MG.

Como fator mesológico de mortalidade natural, acredita-se que a chuva tem exercido efeito negativo elevado na população do bicho-mineiro, provocando morte das lagartas por afogamento no interior da mina (Wolcot, 1947; Amaral, 1953; Avilés, 1991; Pereira et al., 2007). Nantes \& Parra (1977) observaram que o ciclo de desenvolvimento do bicho-mineiro foi mais curto no período chuvoso com elevadas temperaturas; todavia, descartaram a possibilidade de ocorrência do efeito mecânico da água da chuva (Parra, 1985).

Vários trabalhos desenvolvidos em casa de vegetação têm evidenciado que plantas desenvolvidas em condições de estresse hídrico são mais danificadas, propiciando a formação de adultos do bichomineiro com elevada fecundidade e longevidade (Fanton, 1991; Meireles et al., 2001). Brotações novas do cafeeiro podem ser altamente defendidas com emissão de toxinas presentes em folhas do cafeeiro e que provocam distúrbios fisiológicos ou mortalidade de lagartas do bicho-mineiro.
Nesse mesmo período (2000), também se constatou menor número de pupas do bicho-mineiro no sistema orgânico que no convencional. Porém, não se observaram diferenças quanto à predação de lagartas do bicho-mineiro por vespas, que continuou baixa em relação aos índices relatados como ótimos em Minas Gerais (Reis \& Souza, 1998; Reis Jr. et al., 2000). $\mathrm{O}$ controle biológico natural por meio de parasitoides continuou sendo maior no sistema de cultivo orgânico $(25,4 \%)$ que no convencional (12,9\%) (Tabela 3).

Tal como nos últimos meses de 2000, nos primeiros meses de 2001 ocorreram intensas pluviosidades (Figura 1). Isso certamente pode ter afetado a dinâmica populacional do bicho-mineiro, já que nesse período as variáveis que identificaram a injúria do bicho-mineiro foram praticamente nulas. Os primeiros sinais da praga em ambos os sistemas de cultivo ocorreram nos meses de abril a maio quando começa o período frio e seco no sul de Minas Gerais (Tabelas 1 a 3; Figura 1). 
Tabela 3. Parasitismo total de Leucoptera coffeella (\%) por quatro espécies de parasitoides, em função dos sistemas de cultivo do cafeeiro e da época de avaliação. Santo Antônio do Amparo, MG.

\begin{tabular}{|c|c|c|}
\hline \multirow{3}{*}{ Mês } & \multicolumn{2}{|c|}{ Parasitismo total (\%) } \\
\hline & \multicolumn{2}{|c|}{1999} \\
\hline & Convencional & Orgânico \\
\hline Junho & $78,0 \mathrm{aA}$ & $75,5 \mathrm{aA}$ \\
\hline Agosto & $0,0 \mathrm{bA}$ & $80,5 \mathrm{aA}$ \\
\hline \multirow[t]{2}{*}{ Novembro } & $29,2 \mathrm{bA}$ & $40,6 \mathrm{aA}$ \\
\hline & \multicolumn{2}{|c|}{2000} \\
\hline Janeiro & $0,0 \mathrm{bC}$ & $10,7 \mathrm{aC}$ \\
\hline Maio & $0,0 \mathrm{bC}$ & $27,3 \mathrm{aB}$ \\
\hline Agosto & $15,4 \mathrm{bB}$ & $21,1 \mathrm{aB}$ \\
\hline Setembro & $19,4 \mathrm{bB}$ & $23,8 \mathrm{aB}$ \\
\hline Outubro & $16,3 \mathrm{bB}$ & $43,2 \mathrm{aA}$ \\
\hline Novembro & $39,2 \mathrm{bA}$ & $51,7 \mathrm{aA}$ \\
\hline \multirow[t]{2}{*}{ Dezembro } & $0,0 \mathrm{bC}$ & $0,0 \mathrm{aC}$ \\
\hline & \multicolumn{2}{|c|}{2001} \\
\hline Janeiro & $0,0 \mathrm{aB}$ & $0,0 \mathrm{aB}$ \\
\hline Fevereiro & $0,0 \mathrm{aB}$ & $0,0 \mathrm{aB}$ \\
\hline Abril & $10,0 \mathrm{aB}$ & $0,0 \mathrm{aB}$ \\
\hline Maio & 36,0 aA & $0,0 \mathrm{bB}$ \\
\hline Junho & 22,6 aA & $37,2 \mathrm{aA}$ \\
\hline
\end{tabular}

* Médias seguidas pela mesma letra minúscula na linha e maiúscula na coluna não diferem entre si, pelo teste de Scott-Knott $(p \leq 0,05)$.

Aparentemente, o restabelecimento do bicho-mineiro no cafeeiro acontece de forma mais rápida no cultivo convencional em comparação ao orgânico, situação que pode ser atribuída à complexidade das interações do bicho-mineiro com o seu hospedeiro e seus inimigos naturais (predadores, parasitoides e entomopatógenos).

Não foram detectados parasitoides de ovos desse inseto-praga em nenhum dos sistemas de cultivo. As espécies predominantes de parasitoides, obtidas nos dois sistemas de cultivo, foram Orgilus niger Penteado-Dias, 1999; Centistidea striata Penteado-Dias, 1999; Stiropius reticulatus Penteado-Dias, 1999 (Hymenoptera: Braconidae) e Horismenus sp. (Hymenoptera: Eulophidae).

Com base nos dados dos três anos observados, no sistema de cultivo café orgânico ocorreu equilíbrio entre o insetopraga e seus inimigos naturais. Os inimigos naturais e o clima, particularmente a chuva e a temperatura, aparentemente exerceram papel regulador fundamental na dinâmica populacional do bicho-mineiro do cafeeiro. Em sistemas de cultivo convencional os parasitoides podem ser fortemente prejudicados por serem endoparasitoides, sem qualquer possibilidade de escapar do efeito deletério dos produtos fitossanitários.

\section{CONCLUSÕES}

Não se detectou parasitoides de ovos do bicho-mineiro em nenhum dos sistemas de cultivo.

As espécies predominantes de parasitoides em lagartas do bicho-mineiro, nos dois sistemas de cultivo, são Orgilus niger Penteado Dias, 1999; Centistidea 
striata Penteado Dias, 1999; Stiropius reticulatus Penteado Dias, 1999 e Horismenus sp.

\section{REFERÊNCIAS BIBLIOGRÁFICAS}

ABIC. 2009.

ASSOCIAÇÃO

BRASILEIRA DA INDÚSTRIA DE CAFÉ. Notícias ABIC: 24 de maio dia nacional do café. Disponível em: <http://www.abic.com.br/noticias/nota_d ncafe_200509.html $>$. Acesso em: 21 jul. 2009.

AGUIAR-MENEZES, E. L. 2004. Diversidade vegetal: uma estratégia para o manejo de pragas em sistemas sustentáveis de produção agrícola. Seropédica: Embrapa Agrobiologia. 68p. (Documentos, 177).

AGUIAR-MENEZES, E. L.; SANTOS, C. M. A.; RESENDE, A. L. S.; LEAL, M. R.; MENEZES, E. B. 2008. Parasitoides associados às moscas-das-frutas (Diptera: Tephritoidea) em café orgânico com e sem arborização em Valença, RJ, Brasil. Ciência e Agrotecnologia, Lavras, v.32, n.6, p.1824-1831.

AMARAL, E. F. 1953. Considerações sobre o "bicho-mineiro" e sua importância econômica. O Biológico, Campinas, v.19, n.1, p.85-92.

ANTUNES, F. Z. 1986. Caracterização climática do estado de Minas Gerais. Informe Agropecuário, Belo Horizonte, v.12, n.138, p.9-13.

AVILÉS, D. P. 1991. Avaliação das populações do bicho-mineiro do cafeeiro, Perileucoptera coffeella (Lepidoptera: Lyonetiidae) e seus parasitoides e predadores: metodologias de estudo e flutuação estacional. 127p. Dissertação (Mestrado) - Universidade Federal de Viçosa, Viçosa, MG.

ECOLE, C. C.; MORAES, J. C.; VILELA, M. 2010. Suplementos alimentares no manejo do bicho-mineiro e de seus inimigos naturais em cafeeiro convencional adensado. Coffee Science, Lavras, v.5, n.2, p.97-189.

FANTON, C. J. 1991. Efeito do déficit hídrico na biologia do bicho-mineiro do cafeeiro, Perileucoptera coffeella (Lepidoptera: Lyonetiidae). 32p. Dissertação (Mestrado em Entomologia) - Universidade Federal de Viçosa, Viçosa, MG.

FRAGOSO, D. B.; JUSSELINO FILHO, P.; PALLINI FILHO, A.; BADJI, C. A. 2002. Ação de inseticidas organofosforados utilizados no controle de Leucoptera coffeella (Guèr-Ménev.) (Lepidoptera: Lyonetiidae) sobre o ácaro predador Iphiseiodes zuluagai Denmark \& Muma (Acari: Phytoseiidae). Neotropical Entomology, Londrina, v.31, n.3, p.436-467.

GUEDES, R. N. C.; FRAGOSO, D. B. 1999. Resistência a inseticidas: Bases gerais, situação e reflexões sobre o fenômeno em insetos-praga do cafeeiro. In: Encontro sobre produção de café com qualidade, 1., Viçosa p.99-120.

MEIRELES, D. F.; CARVALHO, J. A.; MORAES, J. C. 2001. Avaliação da infestação do bicho-mineiro e do crescimento do cafeeiro submetido a diferentes níveis de déficit hídrico. Ciência e Agrotecnologia, Lavras, v.25, n.2, p.371- 374.

MELO, T. L.; CASTELLANI, M. A.; NACIMENTO, M. L.; MENEZESJUNIOR, A. O.; FERREIRA, G. F. P.; LEMOS, O. L. 2007. Comunidades de parasitoides de Leucoptera coffeella (Guérin-Mèneville \& Perrottet, 1842) (Lepidoptera: Lyonetiidae) em cafeeiros nas regiões oeste e sudeste da Bahia. Ciência e Agrotecnologia, Lavras, v.31, n.4, p.966-972.

NANTES, J. F. D.; PARRA, J. R. P. 1977. Biologia de Perileucoptera coffeella (Guérin-Mènéville, 1842) (Lepidoptera: Lyonetiidae) em três variedades de café 
(Coffea spp.). Anais da Sociedade Entomológica do Brasil, Jaboticabal, v.6, n.1, p.156-163.

PARRA, J. R. P. 1985. Biologia comparada de Perileucoptera coffeella (GuérinMènéville, 1842) (Lepidoptera: Lyonetiidae), visando ao seu zoneamento ecológico no Estado de São Paulo. Revista Brasileira de Entomologia, São Paulo, v.29, n.1, p.45-76.

PARRA, J. R. P.; GONÇALVES, W.; PRECETTI, A. A. C. M. 1981. Flutuação populacional de parasitos e predadores de Perileucoptera coffeella (Guérin-Mènéville, 1842) em três localidades do Estado de São Paulo. Turrialba, San Jose, v.31, n.3, p.357364.

PEREIRA, E. J. G.; PICANÇO, M. C.; BACCI, L.; CRESPO, A. L. B.; GUEDES, R. N. C. 2007. Seasonal mortality factors of the coffee leafminer, Leucoptera coffeella. Bulletin of Entomological Researsh, v.97, n.4, p.421-432.

REIS, P. R.; SOUZA, J. C. 1983. Controle biológico do bicho-mineiro das folhas do cafeeiro. Informe Agropecuário, Belo Horizonte, v.9, n.104, p.16-20.

REIS, P. R.; SOUZA, J. C. 1998. Manejo integrado das pragas do cafeeiro em Minas Gerais. Informe Agropecuário, Belo Horizonte, v.19, n.193, p.17-25.

REIS JR, R.; LIMA, E. R.; VILELA, E. F.; BARROS, R. S. 2000. Method for maintenance of coffee leaves in vitro for mass rearing of Leucoptera coffeella (Guérin-Mèneville) (Lepidoptera: Lyonetiidae). Anais da Sociedade Entomológica do Brasil, Londrina, v.29, n.4, p.849-854.

SCOTT, A. J.; KNOTT, M. A. 1974. A cluster analysis method for grouping means in the analysis of variance. Biometrics, Raleigh, v.30, n.3, p.507512.
SOUZA, J. C.; REIS, P. R.; RIGITANO, R. L. O. 1998. Bicho-mineiro do cafeeiro: biologia, danos e manejo integrado. 2 . ed. Belo Horizonte: EPAMIG, 47p. (EPAMIG. Boletim Técnico, 54)

THEODORO, V. C. A.; GUIMARÃES, R. J.; MOURÃO JÚNIOR, M.; CHAGAS, S. J. R. 2002. Alterações da qualidade de grãos de cafés ( $C$. arábica L.) colhidos no pano e no chão, provenientes de sistemas de manejo orgânico, em conversão e convencional. Revista Brasileira de Armazenamento, Viçosa, Especial Café, n.4, p.38-44.

WOLCOTT, G. N. 1947. A quintessence of sensitivity: the coffee leaf-miner. Jornal de Agricultura Universidade de Porto Rico, Rio Piedras, v.3, n.2, p.215-219. 